\title{
array-CGH revealed gain of Yp11.2 in 49,XXXXY and gain of Xp22.33 in 48,XXYY karyotypes of two rare klinefelter variants
}

\author{
Somprakash Dhangar, Jagdeeshwar Ghatanatti, Babu Rao Vundinti
}

Department of Cytogenetics, National Institute of Immunohaematology (ICMR), K.E.M Hospital campus, Parel, Mumbai, India.

\begin{abstract}
SUMMARY Klinefelter syndrome (KS) variants often share common features with classical syndrome but some of these variants present with a distinct phenotype. The incidence of sex chromosome tetrasomy and pentasomy are very less and generally diagnosed after prepubertal age. The early diagnosis of complex and unclassified syndromes and it's correlation with genotype is necessary for personalized treatment as well as genetic counselling of the affected families. We describe clinical presentation, and genetic diagnosis of two cases of variant KS. Our first case, a 4 year old male child presented with generalized tonic-clonic seizures (GTCSs), delayed milestones and dysmorphic features while case 2, a-21 years old male who had history of seizures and delayed puberty came to our lab for genetic diagnosis. The chromosomal analysis of case 1 and 2 showed 49,XXXXY and 48,XXYY karyotype respectively. The karyotype results were confirmed with fluorescence in situ hybridization (FISH) and array-CGH analysis. The FISH results were found to be consistent with karyotype but the array-CGH results showed the extra gain of region Yp11.2 in case 1 while the extra gain of region Xp22.33 in case 2. The cases were confirmed as variant KS on the basis of additional sex chromosomes and clinical presentation of deteriorated brain development. The present study suggests that the high doses of sex chromosome linked genes including pseudoautosomal region (PAR) caused the abnormal brain development. The combination of molecular techniques should be utilized for the diagnosis of such complex cases to understand the genotype-phenotype correlation and appropriate genetic counseling.
\end{abstract}

Keywords Klinefelter variants, sex chromosome polysomy, pseudoautosomal region, complex psychological behavior, deteriorated brain development

\section{Introduction}

Klinefelter syndrome (KS) variants are rare sex chromosome aneuploidy (SCA), which generally occur less frequently compared to classical KS (1:650 male births) (1-3). Sex chromosome tetrasomy and pentasomy individuals share many features including tall stature, undescended testis and hypergonadotropic hypogonadism. According to the literature the phenotypes in variant $\mathrm{KS}$ cases become more complex as number of sex chromosomes involvement increased (4). The presence of additional sex chromosomes causes an overdose of genes leading to overexpression of protein and affects the normal development of the phenotype. The variant $48, \mathrm{XXYY}$ (tetrasomy) is found to be more common $(1: 18,000-1: 40,000)$ SCA as compared to $49, \mathrm{XXXXY}$ (pentasomy) which is estimated to be present in 1:85000-1:100000 male births (5). The chromosomal aneuploidy occurs through a non-disjunction mechanism in germ cells during gametogenesis or early embryonic development. However the number of extra chromosomes depends on the type of participating gametes and time of the events (6). The 46,XXYY variant mainly results due to fertilization of a normal oocyte with a aneuploid sperm and some times during postzygotic events while 49, XXXXY variant occurs from non- disjunction of the $\mathrm{X}$ chromosome during both meiosis- I and meiosisII stage of oogenesis. Thus an aneuploid oocyte is fertilized with normal sperm $(7,8)$.

The knowledge regarding complex psychological behavior with deteriorated brain development in rare cases of variant KFS are limited and only a few cases are reported in the literature (9). We report genetic analysis of two cases of variant KS present with complex features and abnormal brain development.

\section{Materials and Methods}

Two cases with dysmorphic features with delayed 
milestones were referred to our cytogenetic laboratory for genetic diagnosis. Blood samples were collected in sodium heparin and ethylenediaminetetraacetic acid (EDTA) vacutainers ( $2 \mathrm{~mL}$ in each tube). The heparinized blood samples were used for cytogenetic analysis while EDTA samples were utilized for molecular analysis.

\subsection{Conventional cytogenetics}

The cytogenetic study was carried out by setting up peripheral blood cultures at $37^{\circ} \mathrm{C}$ for $72 \mathrm{hrs}$ according to standard procedures (10). Briefly, the cultures were stimulated with phytohaemagglutinin (PHA) arrested with colchicine $(50 \mu \mathrm{g} / \mathrm{mL})$ and treated with hypotonic solution (KCL 0.56g/100 mL). The cells were fixed in Carnoy's solution (Methanol: Glacial acetic acid; 3:1). The chromosomal preparations obtained were subjected to GTG banding (11). At least 50 metaphases were scored and 10 were karyotyped (approximately 350 band resolution) according to International System of Chromosome Nomenclature 2016 (ISCN 2016) (12). The images were captured with a CCD camera attached to Nikon 90i microscope (Japan) and analysis was done using Applied Spectral Imaging software system (Inc. Carlsbad, USA).

\subsection{Fluorescence in situ hybridization (FISH)}

Metaphases obtained from conventional cytogenetic procedure were utilized for molecular cytogenetic study. Fluorescence in situ hybridization (FISH) was carried out using centromere specific (Vysis, Abbott Life Sciences, USA) probe for X (Cat. No.05J10-033) and Y (Cat. No. 05J08-035) chromosomes.

\section{3. array-CGH}

DNA was extracted from EDTA blood samples using a commercially available DNA kit (QIAamp DNA blood mini kit cat. No. 51104, Qiagen, Germany). Chromosomal microarray analysis (CMA) was performed using CytoPrime microarray. The cutoff filter settings for the CMA test analysis were $1 \mathrm{MB}$ for clinically relevant losses and $2 \mathrm{MB}$ for gains.

\subsection{Case 1}

A 4-year old male child, the first born of a healthy nonconsanguineous couple was referred to our cytogenetics laboratory for chromosomal analysis due to generalized tonic-clonic seizures (GTCSs) episodes, delayed milestones and dysmorphic features. This male patient was born at 8 months of pregnancy (preterm) through lower (uterine) segment caesarean section (LSCS) with moderately low birth weight. The mother of the child had no obvious history of illness or medication during pregnancy and her age was 30 years while the father was 32 years old. On clinical examination, the child had facial dysmorphic features such as slight downward slanting of the eyes with epicanthic folds, hypertelorism, low set ears, large pinnae, and flattened nasal bridge. The speech development was normal while the gross and fine motor development was poor. The proband also had limb anomalies including genu valgum and hyperlaxity of the upper limb. The Social Quotient (SQ) was 75. The magnetic resonance imaging (MRI) scan of the brain revealed demyelization of right parietal sub white matter, mild prominence at lateral and third ventricle with paucity of white matter. Brainstem Evoked Response Audiometric (BERA) test showed normal hearing ability. Ultra sonography (USG) of kidney, ureter and bladder and Barium swallow study revealed normal development. The study was carried out with the consent of the father of the proband.

\subsection{Case 2}

A 21- years old male was referred to our cytogenetic laboratory for chromosomal analysis. He had history of delayed puberty. This male patient was born at full term to non-consanguineous parents.. His mother brought him to the laboratory for the diagnosis of delayed puberty. The mother's age was 31 years while father was 36 years old at the time birth of the affected child. There was no obvious history of illness or medication of mother during pregnancy. The child birth history revealed that this affected patient was born with hypospadias and inguinal hernia and the volume of testis was comparatively low at the time of birth. The child also had history of delayed cry. The child was diagnosed with hydrocele at the age of 3 years and was corrected by surgery. The child had history of delayed puberty and convulsion disorder. During the prepubertal age of proband, mother of the child noticed aggressiveness and poor social behavior with family members. The proband also had history of tremalansness (tremors), which developed after the pubertal age. The clinical examination revealed absence of pubic and axillary hair. The proportion of upper and lower segment was not appropriate. The level of luteinizing hormone (LH) was $21.5 \mathrm{IU} / \mathrm{mL}$ (normal range 2.5-10 $\mathrm{IU} / \mathrm{mL}$ ) while follicular stimulating hormone (FSH) level was $63.0 \mathrm{IU} / \mathrm{mL}$ (normal range 2.5-10 IU/mL). The level of testosterone was very low $(0.27 \mathrm{nmol} / \mathrm{L})$. Thyroid stimulating hormone level was $212 \mu \mathrm{IU} / \mathrm{mL}$ (normal range 0.4- $4.0 \mu \mathrm{IU} / \mathrm{mL}$ ). He also had an elevated level of creatine phosphokinase. The MRI brain of proband revealed periventricular gliotic changes in post parietal periventricular region with left subtle atrophy of right hippocampal tail. The sibling of the proband also had a similar history of medical problems such as protruding tongue, hypertelorism and seizures. The study was carried out with the consent of the parents of the proband. 


\section{Results and Discussion}

The conventional cytogenetic analysis of case 1 revealed 49, XXXXY karyotype while case 2 was detected with 48,XXYY karyotype (Figure 1A and Figure 2A). The karyotypes of parents of both patients were normal. FISH results were consistent with conventional karyotype results and showed four copies of chromosome $\mathrm{X}$ and one copy of chromosome $\mathrm{Y}$ in case 1 while one extra copy of chromosome $\mathrm{X}$ and $\mathrm{Y}$ in case 2 (Figure 1B and Figure 2B). Mosaicism was not observed in both cases.

Further the chromosome gain and breakpoint were confirmed by array-CGH analysis (750K CytoPrime microarray system with Chromosome Analysis Suite software designed by Affymetrix). The array-CGH analysis of case 1 revealed a four copy number gain of entire chromosome X (cytoband Xp22.33-q28) and one extra partial copy number gain of chromosome Y for region Yp11.2 along with entire Y chromosome

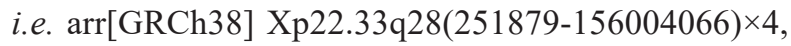
and $\operatorname{arr}[G R C h 38] Y p 11.2(2931110-6204020) \times 2$ (Figure 1C). The array-CGH analysis of case 2 revealed a copy number gain of chromosome $\mathrm{X}$ and $\mathrm{Y}$ i.e. $\operatorname{arr}[\mathrm{GRCh} 38]$

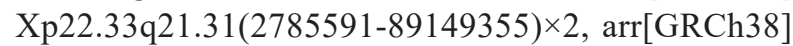

Xq21.31-q28(9110165-155641381) $\times 2$ and $\operatorname{arr}[G R C h 38]$ Yp11.2(2782099-26653790) $\times 2$ respectively. The array$\mathrm{CGH}$ analysis of case 2 also showed a gain at Xp22.33 region, which encompassed 24 genes i.e. $\operatorname{arr}[\mathrm{GRCh} 38]$ Xp22.33(251879-2771493) $\times 4$ (Figure 2C). The arrayCGH analysis of the sibling of case 2 showed a normal result. Apart from the entire intact chromosome the array- CGH detected the extra gain of chromosome Y with accurate break point (Yp11.2) in case 1 and extra gain of chromosomal region Xp22.33 in case 2 which could not be detected by conventional method. The array-CGH result confirmed the diagnosis as variant $\mathrm{KS}$ in both patients with tetrasomy of chromosome $\mathrm{X}$ and disomy (partial) of chromosome $\mathrm{Y}$ in case 1 while tetrasomy (partial) of chromosome $\mathrm{X}$ and disomy of $\mathrm{Y}$ in case 2 .

The variants of KFS are generally defined on the basis of variable number of sex chromosomes. The incidence of KFS variants vary and it depends on the type of chromosome involved. The incidence of variants of KFS is reported to be $1: 18,000$ to $1: 1,000,000$ male births and found to be rarer than classical KS (13). Our literature search revealed lack of information regarding incidence, and genotype phenotype correlation on
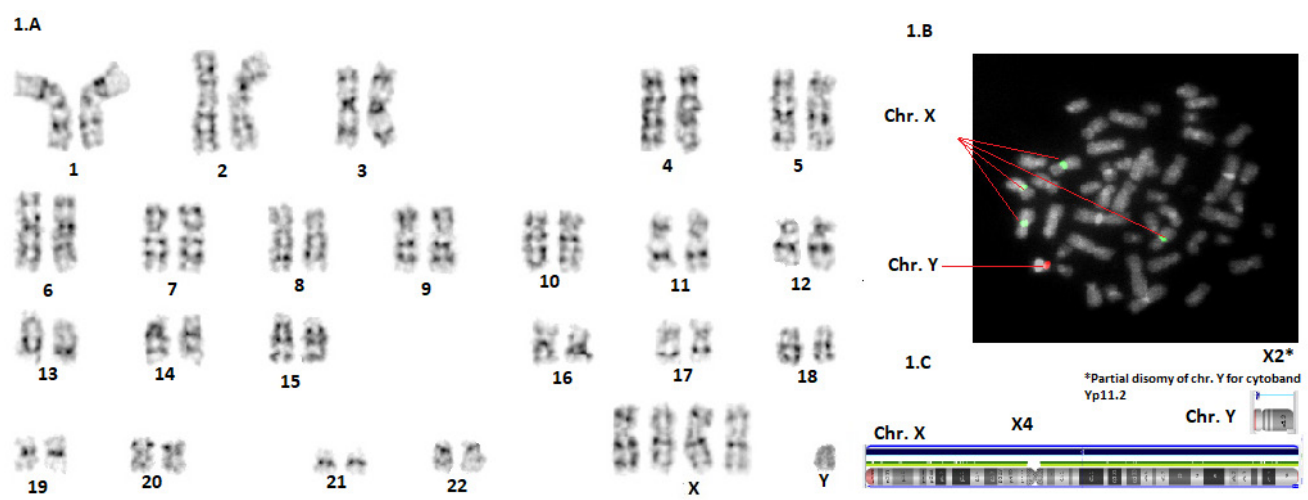

Figure 1. Conventional and molecular cytogenetic analysis of case 1. (A) GTG banded karyotype showing 49,XXXXY karyotype. (B) Tetrasomy of chromosome X (green) and single copy of chromosome Y (red) by FISH. (C) Array- CGH image showing tetrasomy of chromosome $\mathrm{X}$ and partial disomy of chromosome $\mathrm{Y}$ for region Yq11.2.
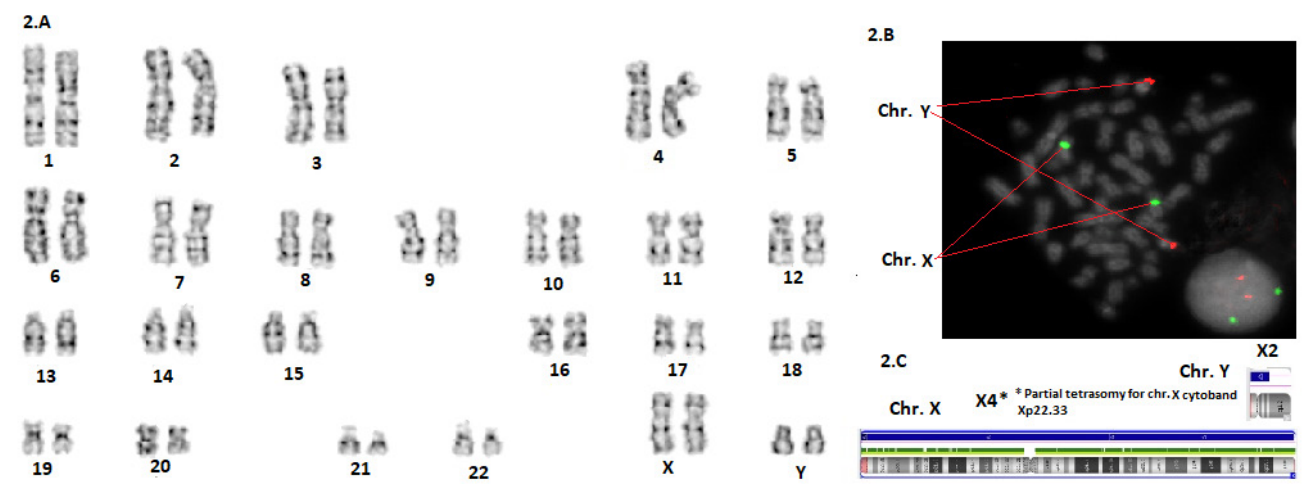

Figure 2. Conventional and molecular cytogenetic analysis of case 2. (A) GTG banded karyotype showing 48,XXYY karyotype. (B) Disomy of X (green) and Y ( red) chromosomes by FISH. (C) Array- CGH image showing partial tetrasomy of chromosome X for region Xp22.33 and disomy of chromosome $\mathrm{Y}$. 
Table 1. Comparison of clinical abnormalities of present cases with reported clinical presentations of SCAs

\begin{tabular}{|c|c|c|c|}
\hline \multirow{2}{*}{ S. No. } & \multirow{2}{*}{ Clinical presentation of sex chromosome polysomy in males ${ }^{*}$} & \multicolumn{2}{|c|}{ Clinical abnormalities observed in present study } \\
\hline & & Case 1 & Case 2 \\
\hline 1 & Hypotonia in childhood & $+($ mild $)$ & - \\
\hline 2 & Hypergonadotropic hypogonadism & + & + \\
\hline 3 & Dental problems & - & - \\
\hline 4 & Asthma/ Reactive airways disease & - & - \\
\hline 5 & Food/ Environmental allergies & - & - \\
\hline 6 & Cardiac malformations & - & - \\
\hline 7 & Intention and postural tremor & - & + \\
\hline 8 & Radioulnarsynostosis/Congenital elbow dislocation & + & - \\
\hline 9 & Congenital hip dysplasia & - & - \\
\hline 10 & Pes planus/ Flat feet & + & + \\
\hline 11 & Club foot & - & - \\
\hline 12 & Inguinal hernia/Undescended testis & - & + \\
\hline 13 & Deep vein thrombosis/ Pulmonary embolism & - & - \\
\hline 14 & Hypothyroidism & - & + \\
\hline 15 & Type II diabetes & - & - \\
\hline 16 & Scoliosis & - & - \\
\hline 17 & Seizure disorder & + & + \\
\hline 18 & Strabismus & + & - \\
\hline 19 & Recurrentotitis & + & - \\
\hline 20 & Gastroesophagealreflux & - & + \\
\hline 21 & Constipation & - & + \\
\hline 22 & Obstructive sleep apnoea & - & - \\
\hline 23 & Osteoporosis & - & - \\
\hline
\end{tabular}

"The clinical presentations mentioned in the above table are not necessary to be present in all cases of sex chromosome aneuploidies (SCAs).

variants of SCA. However the Genetic and rare disease information center (GARD) is one of the important rare genetic disease databases and included these aneuploidies as numeric sex chromosome variations (14). Our extensive literature survey found that different types of KS variants (47,XYY; 48,XXXY; 48,XXYY; 48,XYYY; 49, XXXYY; 49,XXXXY) including copy number variations (CNV's) in sex chromosomes have been reported. However the literature related to CNV's are limited (15). The SCA's occurs due to error in cell division (non-disjunction) during gametogenesis or post zygotic events. In case of mosaic trisomy $(46, \mathrm{XY} /$ $46, \mathrm{XXY})$, the non- disjunction takes place in somatic cells during fetal development $(7,8,16,17)$. The classical features of KS include tall stature with eunuchoid skeleton, pes planus, clinodactyly of fifth finger and primary testicular insufficiency. The small volume of testis is the major clinical presentation in KS individuals, which leads to decreased production of testosterone and ultimately affects male sexual development. However, the clinical presentation of KS variants differs from case To case and affects different systems of the body (Table 1) (18). Despite multiple reports on effects of SCA and disease severity, the knowledge of abnormal brain development is limited $(19,20)$. In our study we have identified aneuploidy of sex chromosomes in both cases, and it is evident that these anomalies occur denovo in the index cases because parental cytogenetic analysis revealed normal karyotypes. The non- disjunction mechanism taking place during gametogenesis in one of the parents may be the reason for the aneuploidy in the proband. In addition, the microarray analysis showed duplication of pseudoautosomal regions (PAR) PAR 1 and PAR 2 on the sex chromosomes. The abnormal crossing over taking place in the sex chromosome during gametogenesis involving the PAR1 and PAR 2 regions could be the reason for Copy number Variations (21).

In the present study, both cases were diagnosed as Variant KS with novel karyotype. In Case 1, the proband was diagnosed with 49, XXXXY karyotype. The proband also had partial disomy of chromosome $\mathrm{Y}$ for region Yp 11.2. Apart from the classical features (undescended testis, genu valgum and hyperlaxity of the upper limb) the proband also had brain anomalies like demyelination of the right parietal sub white matter, and mild prominence at lateral and third ventricle with paucity of white matter. The gene dosage of sex chromosomes were found to be associated with learning disabilities and language development, because the genes lying on the sex chromosome have a crucial role in brain development and language pathways (22).

The 48,XXYY karyotype is an uncommon chromosomal abnormality in humans. These anomalies occur due to non- disjunction of homologous chromosome during gametogenesis. The individual with 48,XXYY karyotype generally presented with tall stature, which became more significant during adolescence (18). In the present study, the proband (case 2) was diagnosed with 48,XXYY karyotype and also had partial tetrasomy $\mathrm{X}$ for the PAR region. The proband presented with tall stature and detoriated brain 
development. Though Attention Deficit Hyperactivity Disorders (ADHD) are more common (70\%) in $48, \mathrm{XXYY}$ cases, evidence related to etiological genetic factors are limited. In our study we have identified brain anomalies in both cases, which are found to be associated with genotype. In our first case MRI study revealed demyelination of right parietal sub white matter, mild prominence at lateral and third ventricle with paucity of white matter while the second case shows periventricular gliotic changes in post parietal periventricular region with left subtle atrophy of right hippocampal tail. Similar clinical features also reported as case reports suggested that the gene dosage of $\mathrm{X}$ chromosome plays an important role in development of white matter tracts and ventricles of the brain and also reduces brain volume $(9,22)$. Our CMA (CytoPrime microarray) analysis identified copy number gain at position Xp22.33 in case two, and the genes lying on this chromosomal region (Xp22.33) are found to be associated with ADHD and autism spectrum disorder, however pathogenicity is not yet clear (23). In case 1 apart from 49,XXXY Karyotype, our CMA analysis also identified a copy number gain at cytoband Yp11.2, which encompasses five genes ( $Z F Y$, ZFY-AS1, LINC00278, TGIF2LY, PCDH11Y) about $3.2 \mathrm{MB}$ in size. According to the literature, the gene $P C D H 11 Y$ (paralogue of $P C D H 11 X$ ) has an important role in cell- cell recognition during development of the central nervous system which might play a role in brain development and reduced brain volume (24). Moreover the genes present in pesudoautosomal region expressed (2.0 - fold in 48,XXYY, 48XXXY or 2.5- fold in $49, \mathrm{XXXXY}$ ) more as compared to classical KFS cases because these genes can escape $\mathrm{X}$-inactivation. The over expression of these genes are found to be associated with different developmental problems $(25,26)$.

In conclusion, the diagnosis of variants of clinical syndromes is important to understand the genetic nature and phenotype presentation. Though chromosomal aneuploidies are identified with routine cytogenetic investigation, the microarray analysis helps in identification of submicroscopic losses and gains, which greatly helps in genotype-phenotype correlation and appropriate genetic counseling.

\section{Acknowledgements}

The study was carried out with an Institutional core grant.

\section{References}

1. Evans J A, de von Flindt R, Greenberg C et al. A cytogenetic survey of 14,069 new born infants, IV, further follow-up on the children with sex chromosome anomalies. In: Birth Defects: Original Article Series (Stewart D, Ed.). Alan R. Liss, Inc, New York, USA,
1982; pp.169-184.

2. Fruhmesser A, Kotzot D. Chromosomal variants in klinefelter syndrome. Sex Dev. 2011; 5:109-123.

3. Asirvatham AR, Pavithran PV, Pankaj A, Bhavani N, Menon U, Menon A, Abraham N, Nair V, Kumar H, Thampi MV. Klinefelter syndrome: clinical spectrum based on 44 consecutive cases from a South Indian Tertiary Care Center. Indian J Endocr Metab. 2019; 23:263-266.

4. Radicioni AF, Ferlin A, Balercia G, Pasquali D, Vignozzi L, Maggi M, Foresta C, Lenzi A. Consensus statement on diagnosis and clinical management of Klinefelter syndrome. J Endocrinol Invest. 2010; 33:839-850.

5. Kleczkowska A, Fryns J, Van den Berghe H. X-chromosome polysomy in the male. Hum Genet. 1988; 80:16-22.

6. Maiburg M, Repping S, Giltay J. The genetic origin of Klinefelter syndrome and its effect on spermatogenesis. Fertil Steril. 2012; 98:253-260.

7. Hassold T, Hunt P. To err (meiotically) is human: the genesis of human aneuploidy. Nat Rev Genet. 2001; 2:280-291.

8. Hassold T, Hall H, Hunt P. The origin of human aneuploidy: where we have been, where we are going. Hum Mol Genet. 2007; 16:R203-208.

9. Blumenthal JD, Baker EH, Lee NR, Wade B, Clasen LS, Lenroot RK, Giedd JN. Brain morphological abnormalities in $49, \mathrm{XXXXY}$ syndrome: a pediatric magnetic resonance imaging study. Neuroimage Clin. 2013; 2:197-203.

10. Moorhead PS, Nowell PC, Mellman WJ, Battips DM, Hungerford DA. Chromosome preparations of leukocytes cultured from human peripheral blood. Exp Cell Res.1960; 20:613-616.

11. Seabright M: A rapid banding technique for human chromosomes. Lancet.1971; 2:971-972.

12. McGowan-Jordan J, Simons A, Schmid M, (eds.). An international system for human cytogenomic nomenclature. Reprint of Cytogenetic and Genome Res, S Karger, Basel, New York, 2016, 149:1-2.

13. Linden MG, Bender BG, Robinson A. Sex chromosome tetrasomy and pentasomy. Pediatrics. 1995; 96:672-682.

14. The Genetic and Rare Diseases Information Center (GARD). Numeric-sex-chromosome-variations. https:// rarediseases.info.nih.gov/diseases/11919/numeric-sexchromosome-variations (accessed January 5, 2020).

15. Alberto F, Maria SR, Vanna P, Riccardo S, Nicola C, Carlo F. Copy number variations in the $\mathrm{X}$ chromosome of Klinefelter syndrome. Endocrine Abstracts 2015; 37 EP221.

16. Rinaldi A, Archidiacono N, Rocchi M, Filippi G. Additional pedigree supporting the frequent origin of XXYY from consecutive meiotic non-disjunction in paternal gametogenesis. J Med Genet. 1979; 16:225-226.

17. Lorda-Sanchez I, Binkert F, Hinkel K G, Moser H, Rosenkranz W, Maechler M, Schinzel A. Uniparental origin of sex chromosome polysomies. Hum Hered. 1992; 42:193-197.

18. Tartaglia N, Davis S, Hench A, Nimishakavi S, Beauregard R, Reynolds A, Fenton L, Albrecht L, Ross J, Visootsak J, Hansen R, Hagerman R. A new look at XXYY syndrome: Medical and psychological features. Am J Med Genet Part A. 2008; 146A:1509-1522.

19. Galasso C, Arpino C, Fabbri F, Curatolo P. Neurologic Aspects of 49,XXXXY Syndrome. J Child Neurol. 2003; 
18:501-504.

20. Haeusler G, Frisch H, Guchev Z, Hadziselimovic F, Neuhold A, Vormittag W. Hypoplasia of the corpus callosum and growth hormone deficiency in the XXXXY syndrome. Am J Med Genet. 1992; 44:230-232.

21. Helena MA, Morris BJ. The human pseudoautosomal region (PAR): origin, function and future. Curr Genomics. 2007; 8:129-136.

22. Dhakar MB, Ilyas M, Jeong JW, Behen ME, Chugani HT. Frontal aslant tract abnormality on diffusion tensor imaging in an aphasic patient with 49, XXXXY syndrome. Pediatr Neurol. 2016; 55:64-67.

23. Green T, Flash S, Reiss AL. Sex differences in psychiatric disorders: what we can learn from sex chromosome aneuploidies. Neuropsychopharmacology. 2019; 44:9-21.

24. Shamsi MB, Kumar K, Dada R. Genetic and epigenetic factors: role in male infertility. Indian J Urol. 2011; 27:110-120.

25. Vawter MP, Harvey PD, and De Lisi LE. Dysregulation of X-linked gene expression in Klinefelter's syndrome and association with verbal cognition. Am J Med Genet.
2007; 144B:728-734.

26. Geschwind DH, Gregg J, Boone K, Karrim J, Pawlikowska- Haddal A, Rao E, Ellison J, Ciccodicola A, D'Urso M, Woods R, Rappold GA, Swerdloff R, Nelson SF. Klinefelter's syndrome as a model of anomalous cerebral laterality: Testing gene dosage in the $\mathrm{X}$ chromosome pseudoautosomal region using a DNA microarray. Dev Genet. 1998; 23:215-229.

Received March 4, 2020; Revised May 19, 2020; Accepted May 30, 2020

\section{*Address correspondence to:}

Babu Rao Vundinti, Department of Cytogenetics, National Institute of Immunohaematology (ICMR), 13th floor, new multistoried building, K.E.M Hospital campus, Parel, Mumbai-400012, India

E-mail: vbaburao@hotmail.com

Released online in J-STAGE as advance publication June 27, 2020. 